\title{
Triple arrays from difference sets
}

\author{
Tomas Nilson $^{1}$ (iD) | Peter J. Cameron ${ }^{2}$
}

\author{
${ }^{1}$ Department of Science Education and Mathe- \\ matics, Mid Sweden University, Sweden \\ ${ }^{2}$ School of Mathematics and Statistics, Univer- \\ sity of St Andrews, Scotland \\ Correspondence \\ Tomas Nilson, Department of Sci- \\ ence Education and Mathematics, \\ Mid Sweden University, Sweden. \\ Email: tomas.nilson@miun.se
}

\begin{abstract}
This paper addresses the question of whether triple arrays can be constructed from Youden squares developed from difference sets. We prove that if the difference set is abelian, then having -1 as multiplier is both a necessary and sufficient condition for the construction to work. Using this, we are able to give a new infinite family of triple arrays. We use an alternative version of the construction to analyze the case of non-abelian difference sets, for which we prove a sufficient condition for giving triple arrays. We do a computer search for such non-abelian difference sets, but have not found any examples satisfying the given condition.

\section{K E Y W O R D S}

block design, difference set, triple array, youden square
\end{abstract}

\section{1 | INTRODUCTION}

Agrawal [1] considered a type of row-column design that now goes by the name of "triple array." He observed that such an array implies the existence of a symmetric-balanced incomplete block design, and asked whether triple arrays can be constructed from such designs. The statement that this can always be done, with exception for trivial and the smallest nontrivial design, is known as Agrawal's conjecture.

Definition 1.1. A triple array is an $r \times c$ array on $v$ symbols arranged so that no symbol occurs more than once in any row or column, and satisfies the following four conditions:

TA1. Each symbol occurs $k$ times (equireplicate).

TA2. Any two distinct rows contain $\lambda_{r r}$ common symbols.

TA3. Any two distinct columns contain $\lambda_{c c}$ common symbols.

TA4. Any row and column contain $\lambda_{r c}$ common symbols.

For a triple array we use the notation $T A\left(v, k, \lambda_{r r}, \lambda_{c c}, \lambda_{r c}: r \times c\right)$. An array as above that satisfies conditions TA1-TA3 is called a double array with notation $D A\left(v, k, \lambda_{r r}, \lambda_{c c}: r \times c\right)$, and a double array that does not satisfy TA4 is called a proper double array. 
If we interchange the roles of columns and symbols in a triple array, we get what is called the $R L$ form of a triple array, (short for Row-Letter form, sometimes used in the statistics literature). It is an incomplete $r \times v$ array, with no repetition in any row or column, satisfying the following four conditions, corresponding to the above conditions TA1-TA4:

RTA1. Every column has $k$ occupied cells.

RTA2. For any pair of rows, there are $\lambda_{r r}$ columns in which both rows are occupied.

RTA3. Every pair of symbols must occur together in $\lambda_{c c}$ columns.

RTA4. The columns with occupied cells in row $i$ must contain every symbol exactly $\lambda_{r c}$ times, for all $i, 1 \leq i \leq r$.

Correspondingly, an array as above satisfying RTA1-RTA3 is the RL-form of a double array.

Triple arrays satisfy the following inequality.

Theorem 1.2. [14] Any TA( $\left.v, k, \lambda_{r r}, \lambda_{c c}, \lambda_{r c}: r \times c\right)$ satisfies $v \geq r+c-1$.

Here we consider the extremal case that is relevant for Agrawal's conjecture and the relation to symmetric designs, and for which there are many examples. In the nonextremal case there is only one example known, a $T A(35,3,5,1,3: 7 \times 15)[14]$.

Definition 1.3. A $(v, k, \lambda)$-balanced incomplete block design (BIBD) is a pair $(V, \mathcal{B})$ where $V$ is a $v$-set and $\mathcal{B}$ is a collection of $b$ proper $k$-subsets of $V$ called blocks such that each element of $V$ is contained in exactly $r$ blocks and any 2 -subset of $V$ is contained in exactly $\lambda$ blocks. A BIBD where $v=b$ is said to be symmetric and is abbreviated as SBIBD.

Note that, in the combinatorial literature, it is common to refer to a BIBD as a 2-design, and a SBIBD as a symmetric (or square) 2-design.

Remark 1.4. Another, but equivalent way to define a double array is to say that rows and symbols form a BIBD, and that columns and symbols form a BIBD. Moreover, condition TA4 is often called adjusted orthogonality.

Agrawal conjectured a construction of triple arrays from SBIBDs, which we now state, together with its converse (which is true).

Conjecture 1.5. [1] If there is a $\left(v+1, r, \lambda_{c c}\right)-S B I B D$ with $r-\lambda_{c c}>2$, then there is a $T A\left(v, k, \lambda_{r r}, \lambda_{c c}, \lambda_{r c}: r \times c\right)$ with $v=r+c-1$.

The condition $r-\lambda_{c c}>2$ excludes the Fano plane and its complement, for which it is known that no triple array exists.

Theorem 1.6. [14] If there is a $T A\left(v, k, \lambda_{r r}, \lambda_{c c}, \lambda_{r c}: r \times c\right)$ with $v=r+c-1$, then there is $a(v+$ $\left.1, r, \lambda_{c c}\right)-S B I B D$.

Agrawal [1] gave a construction method from which many examples have been constructed, but for which one step has not been proven and has to be performed by trial and error. Besides this, only one infinite family called Paley triple arrays has been proven to exist. This has been done to different degrees by Seberry [19], Street [21], Bagchi [2], and Preece et al. [17], and can be summarized as follows.

Theorem 1.7. Let $q \geq 5$ be an odd prime power. Then there exists a $q \times(q+1)$ triple array.

We will use a construction method that starts from Youden squares. 
Definition 1.8. Let $V$ be a set of $v$ symbols. A $k \times v$ Youden square is a $k \times v$ array satisfying

YS1. every symbol of $V$ occurs exactly once in each row, and

YS2. the symbols in each column constitute a $k$-subset of $V$, and the $v$ such subsets constitute the blocks of a $(v, k, \lambda)$-SBIBD.

This construction method, suggested by [18], starts from a Youden square and goes directly to the RL-form of a triple array, thereby avoiding the unproven step of Agrawal's method. However, this places strong demands on the structure of the Youden square, and it was shown in [23] that this method is not even guaranteed to give a double array. But in [16] the usability of this method was further studied, also with some positive results. That a Youden square can always be constructed from a SBIBD was proven by [20]. But the most straightforward way to construct a Youden square is to develop it from a difference set.

In this paper, we investigate if there are difference sets which, if developed to Youden squares, give triple arrays by the suggested construction. The rest of the paper is structured as follows: In Section 2, we give preliminary definitions and results about difference sets. In Section 3, we present the construction and prove that having -1 as a multiplier is both a necessary and sufficient condition for an abelian difference set to give a triple array. In Section 4, we present known results about existence for such difference sets and give parameters for a new infinite family of triple arrays. In Section 5, we present a direct version of the construction and formulate a more general condition for a difference set to give a triple array, suitable for studies of non-abelian sets. Finally, in Section 6, we analyze the non-abelian case. We show by a computer search on small groups that having -1 as weak multiplier, or having a reversible translate, are not sufficient conditions for such a set to give a triple array.

\section{2 | PRELIMINARIES}

We recall some facts from the theory of difference sets.

Definition 2.1. Let $G$ be a finite multiplicative group of order $v$, and $D$ a $k$-subset of $G$. Then $D$ is called a $(v, k, \lambda)$-difference set if any nonidentity element of $G$ can be written in exactly $\lambda$ ways as $x y^{-1}$ where $x$ and $y$ are in $D$. The order of a difference set is the integer $n=k-\lambda$ and $D$ is called nontrivial if $n>1$. We say that $D$ is cyclic or abelian if $G$ is.

Unless stated otherwise, the term "difference set" will here always refer to a nontrivial difference set. Given a difference set, we can find many other related difference sets.

Theorem 2.2 (cf. [15]). Let $D$ be a $(v, k, \lambda)$-difference set in $G$. Then its complement $\bar{D}=G \backslash D$ is a difference set in $G$.

Theorem 2.3 (cf. [15]). Let $D \subset G$ be a $(v, k, \lambda)$-difference set.

1. For $g \in G$, both $g D$ and $D g$ are $(v, k, \lambda)$-difference sets.

2. Let $\alpha$ be an automorphism of $G$. Then $\alpha(D)$ is a $(v, k, \lambda)$-difference set.

For parameters we have the following fundamental identity.

Theorem 2.4 (cf. [15]). Let $D$ be a $(v, k, \lambda)$-difference set in $G$. Then $k(k-1)=\lambda(v-1)$.

We will need the notion of reversible difference sets. 
Definition 2.5. Let $S$ be a subset of a group $G$. Then $S^{(-1)}$ is the set of inverses of the elements of $S$, that is $S^{(-1)}=\left\{x^{-1}: x \in S\right\}$.

Definition 2.6. A difference set $D$ is called reversible if $D=D^{(-1)}$.

Difference sets can be used to construct SBIBDs.

Definition 2.7. Let $X$ be a subset of a finite group $G$. For any $g \in G$, define $X g=\{x g: x \in X\}$. We call any set $X g$ a right translate of $X$ and define the development of $X$, denoted $\operatorname{dev}(X)$, to be the collection of all right translates of $X$.

Theorem 2.8 (cf. [15]). Let $D \subset G$ be a $(v, k, \lambda)$-difference set. Then $(G, \operatorname{dev}(D))$ is a $(v, k, \lambda)$-SBIBD.

Studying difference sets often means dealing with multisets, and then the following algebraic structure can be useful.

Definition 2.9. Let $G$ be a finite multiplicative group. The integral group ring $\mathbb{Z} G$ consists of formal sums $\sum_{g \in G} a_{g} g$ where $a_{g} \in \mathbb{Z}$. Addition and multiplication are defined as follows:

$$
\begin{gathered}
\sum_{g \in G} a_{g} g+\sum_{g \in G} b_{g} g=\sum_{g \in G}\left(a_{g}+b_{g}\right) g \\
\left(\sum_{f \in G} a_{f} f\right)\left(\sum_{g \in G} b_{g} g\right)=\sum_{h \in G}\left(\sum_{f g=h \in G} a_{f} b_{g}\right) h .
\end{gathered}
$$

Let $S \subseteq G$, then it is a standard convention to abuse notation and write also the corresponding group ring element as $S$, that is $S:=\sum_{g \in S} g$. In $\mathbb{Z} G$, if $A=\sum_{g \in G} a_{g} g$ and $t$ any integer, then we define $A^{(t)}:=\sum_{g \in G} a_{g} g^{t}$. In particular, $A^{(-1)}=\sum_{g \in G} a_{g} g^{-1}$. A useful notation concerning cardinality is $|A|=\sum_{g \in G} a_{g}$. We write the identity in $G$ as $1_{G}$ to distinguish it from the integer 1 . Further, $\mathbb{Z} G$ is a ring with identity $1_{G}$ and is commutative if and only if $G$ is abelian.

Theorem 2.10 (cf. [15]). Let $D$ be a nonempty proper subset of a group $G$ with $|D|=k$ and $|G|=v$. Then $D$ is a $(v, k, \lambda)$-difference set of order $n$ if and only if

$$
D D^{(-1)}=n 1_{G}+\lambda G
$$

holds in $\mathbb{Z} G$.

Definition 2.11. Let $D$ be a difference set in a group $G$. An automorphism $\phi$ of $G$ is called a multiplier of $D$ if $\phi(D)=a D b$ for some $a, b \in G$. If $a=1$, then $\phi$ is called a right multiplier. If $G$ is abelian and $\phi$ is of the form $\phi_{t}: x \mapsto x^{t}$ for some integer $t$, then $\phi$ is called a numerical multiplier. It is common practice to abuse terminology and call $t$ itself a numerical multiplier.

For $\phi_{t}: x \mapsto x^{t}$ we write $\phi_{t}(D)=D^{(t)}=\left\{x^{t}: x \in D\right\}$. We will take particular interest in the inverse mapping $x \mapsto x^{-1}$. It is an automorphism of every abelian group but quite rare as multiplier. In non-abelian groups this mapping is not a homomorphism so it cannot be a multiplier, but is called a weak multiplier if $D^{(-1)}$ is a right translate of $D$.

Theorem 2.12 (cf. [4]). Let $D$ be a $(v, k, \lambda)$-difference set in the abelian group $G$. Then there exists a translate of $D$ that is fixed by every numerical multiplier of $D$.

Hence, -1 is a multiplier of $D$ if and only if $D$ has a reversible translate. Also, note that if -1 is a multiplier of $D$, then it also a multiplier of every translate of $D$. To see this, suppose $(D g)^{(-1)}=D g$. Then $g^{-1} D^{(-1)}=D g$, so $D^{(-1)}=D g^{2}$. 
Finally, we will need the following two results for calculating parameters in triple arrays.

Lemma 2.13. [13] Suppose $\mathcal{A}$ is a $T A\left(v, k, \lambda_{r r} . \lambda_{c c}, \lambda_{r c}: r \times c\right)$ with $v=r+c-1$. Then $\lambda_{r c}=r-$ $\lambda_{c c}$.

Corollary 2.14. [13] When $v=r+c-1$ every triple array is a $T A(v, k, c-k, r-k, k: r \times c)$.

\section{3 | TRIPLE ARRAYS FROM ABELIAN DIFFERENCE SETS}

In this section, we construct triple arrays from Youden squares developed from abelian difference sets admitting -1 as a multiplier, and prove that this is both a necessary and sufficient condition for the construction to work. By Theorem 2.8 it is straightforward to construct a Youden square from a difference set.

Construction 3.1. Let $D$ be a $(v, k, \lambda)$-difference set in a finite multiplicative group $G$. Let $Y$ be a $k \times v$ array where the columns are indexed by the elements $j \in G$. Write the elements of $D$ in an arbitrary order in column 1 , and let each row be indexed by the element $i \in D$ in column 1 . Then, take $Y(i, j)=i j$, where $Y(i, j)$ denotes the element in position $(i, j)$ in $Y$.

From a Youden square we construct the RL-form of an array as suggested in [18].

Construction 3.2. Let $Y$ be a Youden square, and $C$ a column in $Y$. The array $Y^{[C]}$ is produced from $Y$ by deleting $C$ from $Y$ together with all the symbols in $C$.

As mentioned before, applying Construction 3.2 to any Youden square $Y$ does not even guarantee that we get the RL-form of a double array. But here $Y$ is developed from a difference set.

Theorem 3.3. Let $D$ be $a(v, k, \lambda)$-difference set in a group $G$. Then there is a $k \times(v-k)$ double array.

Proof. Let $Y^{[C]}$ be an array from Construction 3.2 based on a Youden square $Y$ from Construction 3.1. Then this theorem consists of two results already known put together for constructing the RL-form of a double array. In [16], RTA1 and RTA3 was proven in Proposition 2 for any Youden square $Y$, and in Proposition 5 RTA2 was proven in the special case when $Y$ is constructed from a difference set, without any assumption of being abelian and to hold regardless of the choice of column $C$ for $Y^{[C]}$ in Construction 3.2. Here we write $G$ multiplicatively, whereas it was written additively in [16]. Hence, from $D$ we can construct an RL-form of a double array and thereby a double array with $|D|=k$ rows and $|G|-|D|=v-k$ columns.

Theorem 3.4. Let $D$ be a $(v, k, \lambda)$-difference set in an abelian group $G$ that admits -1 as a multiplier. Then there is a $k \times(v-k)$ triple array.

Proof. Let $Y$ be a Youden square obtained from Construction 3.1 when applied to a difference set such as above. We will prove that applying Construction 3.2 to any column $C$ of $Y$ gives a $Y^{[C]}$ that is the RL-form of a triple array. We already have RTA1, RTA2, and RTA3 by Theorem 3.3 and what remains is to prove RTA4.

That a difference set with multiplier -1 has a reversible translate is given by Lemma 2.12. So, suppose $Y$ is constructed from a difference set $D g$ with reversible translate $D$. Take an element $x \in D g$. We will prove that the columns in $Y$ that have elements from $G \backslash D g$ in row $x$ together contain exactly $n=k-\lambda$ copies of each element in $G \backslash D g$.

We partition $G$ into $L_{x}$ and $R_{x}$ such that $L_{x}$ is the set of column indices for columns having an element of $D g$ in row $x$, and $R_{x}$ consists of the remaining column indices. If wanted, the columns of $Y$ could be permuted as in Figure 1 . 


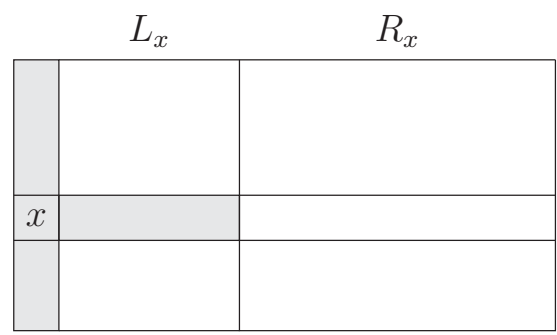

F I G U R E 1 A Youden square where the shaded part of row $x$ and the first column both have support $D g$.

Note that $Y$ can be regarded as part of a multiplication table for $G$ and that in $\mathbb{Z} G$ the product $D g R_{x}$ represents a multiset consisting of the elements in the columns with indices in $R_{x}$, counted with multiplicity. First we note that $x=d g$ for some $d \in D$ and that $x L_{x}=D g$, so

$$
L_{x}=x^{-1} D g=(d g)^{-1} D g=g^{-1} d^{-1} D g=D d^{-1} \text {. }
$$

We will now determine the multiset $D g R_{x}$.

$$
D g R_{x}=D g\left(G-L_{x}\right)=D g G-D g L_{x}=D g G-D g D d^{-1}=D g G-D D g d^{-1}
$$

We note that $D g G=|D g| G=k G$ and since $D$ is reversible we can make use of Theorem 2.10 to write

$$
\begin{aligned}
D g G-D D g d^{-1} & =k G-\left(n 1_{G}+\lambda G\right) g d^{-1}=k G-n g d^{-1}-\lambda G g d^{-1} \\
& =k G-n g d^{-1}-\lambda G=(k-\lambda) G-n g d^{-1}=n G-n g d^{-1}
\end{aligned}
$$

where $g d^{-1} \in D g$.

Hence, we have proved that every element in the columns indexed by $R_{x}$ contain each element of $G \backslash D g$ exactly $n=k-\lambda$ times. Since $x$ is an arbitrary element of $D g$ this holds for any $x \in D g$ thereby proving RTA4 for $Y^{[C]}$ where $D g$ is the support of $C$. And, because $g \in G$ was arbitrary, the proof holds with respect to any column $C$ of $Y$.

And now we will prove the converse, that if a Youden square constructed from an abelian difference set $D$ gives a triple array, then $D$ admits -1 as a multiplier.

For convenience we let $Y_{x}^{[C]}$ denote the array consisting of all columns of $Y^{[C]}$ where row $x \in$ $\operatorname{supp}(C)$ has elements in $G \backslash \operatorname{supp}(C)$.

Lemma 3.5. Let $D$ be a nonreversible abelian difference set that via Construction 3.1 gives a Youden square from which Construction 3.2 gives the RL form of a triple array. Then $\left|D \cap D^{(-1)}\right|=\lambda$.

Proof. Suppose $D$ gives the RL-form of a triple array when applying Construction 3.1 and Construction 3.2, but that $D$ is not reversible. Then there is an $x \in D$ such that $x^{-1} \in \bar{D}$ and we claim that $x^{-1}$ occurs $\left|\bar{D} \cap D^{(-1)}\right|$ times in $Y_{x}^{[C]}$.

Let $y \in D$ such that $y^{-1} \in \bar{D}, x$ and $y$ not necessarily distinct. Any column $z$ where $x z=y^{-1}$ is active in $Y_{x}^{[C]}$, but then we also have $y z=x^{-1}$. Hence, $x^{-1}$ occurs at least $\left|\bar{D} \cap D^{(-1)}\right|$ times in $Y_{x}^{[C]}$. To see that $x^{-1}$ does not occur more times in $Y_{x}^{[C]}$ we assume that $y^{-1} \in D$ and $y w=x^{-1}$. But then $x w=y^{-1}$ so column $w$ is not active in $Y_{x}^{[C]}$ that proves the claim. 
Now, since RTA4 tells us that every element of $\bar{D}$ occur $\lambda_{r c}$ times in $Y_{x}^{[C]}$ and Theorem 1.6 together with Lemma 2.13 give $\lambda_{r c}=k-\lambda$ we have

$$
\lambda_{r c}=k-\lambda=\left|\bar{D} \cap D^{(-1)}\right|=|D|-\left|D \cap D^{(-1)}\right| .
$$

Hence, $\left|D \cap D^{(-1)}\right|=\lambda$.

Lemma 3.6. Let $D$ be a difference set in an abelian group $G$. Then

$$
\left|D g \cap(D g)^{(-1)}\right| \in\{|D|, \lambda\} \quad \text { for all } g \in G
$$

if and only if $\mathbf{D}$ admits -1 as a multiplier.

Proof. Suppose $D$ admits -1 as a multiplier. Then -1 is also a multipler of every translate, so for each $g \in G$ we have $\left|D g \cap(D g)^{(-1)}\right|=\lambda$ or $\left|D g \cap(D g)^{(-1)}\right|=|D|$ since we know by Lemma 2.12 that there is a least one translate $D g$ fixed by the multiplier -1 .

Now, suppose $\left|D g \cap(D g)^{(-1)}\right| \in\{|D|, \lambda\}$ for all $g \in G$ and that -1 is not a multiplier of $D$. So there is no $g \in G$ such that $\left|D g \cap(D g)^{(-1)}\right|=|D|$ that means that $\left|D g \cap(D g)^{(-1)}\right|=\lambda$ for all $g \in G$. Hence, $d_{i} g=g^{-1} d_{j}^{-1}$ or equivalentely $d_{i} d_{j}=g^{-2}$ has exactly $\lambda$ solutions of pairs $d_{i}, d_{j} \in D$ for each $g \in G$.

This would be $\lambda|G|$ elements $g^{-2}$ in total when counted with multiplicity. We compare this with all elements $d_{i} d_{j}$ counted with multiplicity that is given by $|D D|$ in $\mathbb{Z} G$. For $(v, k, \lambda)$-difference sets we have the identity $\lambda(v-1)=k(k-1)$ given in Theorem 2.4 and can write

$$
|D D|=k^{2}>|\lambda G|=\lambda v=k^{2}-(k-\lambda)=k^{2}-n .
$$

So, there is a $g^{-2} \in G$ for which there are more than $\lambda$ solutions of $d_{i} d_{j}=g^{-2}$. And since $|D|$ is the only available number besides $\lambda$ we know that there is a reversible translate that means that -1 is a multiplier.

Theorem 3.7. Let $D$ be an abelian difference set that via Construction 3.1 gives a Youden square from which Construction 3.2 gives the RL form of a triple array, then $D$ admits -1 as a multiplier.

Proof. By Lemma 3.5 we know that a nonreversible abelian difference set $D$ that gives the RL-form of a triple array through Construction 3.1 and Construction 3.2 must satisfy $\left|D \cap D^{(-1)}\right|=\lambda$. But Lemma 3.6 tells us that it is not possible that $\left|(D g) \cap(D g)^{(-1)}\right|=\lambda$ for all $g \in G$. Hence, there exists some $g \in G$ such that $D g$ is reversible that proves that -1 is a multiplier of $D$.

\section{4 | EXISTENCE}

Here we consider abelian difference sets having multiplier -1, or we can say being reversible because of Lemma 2.12. An overview of the theory can be found in [4], here we only cover enough for answering questions relevant for triple arrays. We give all small such difference sets as examples and prove the existence of an infinite family of triple arrays.

Theorem 4.1 (cf. [4]). Let $D$ be a reversible $(v, k, \lambda)$-difference set in an abelian group, then $v$ and $\lambda$ are even and $n$ is a square. Moreover, $v$ and $n$ have the same odd prime divisors.

We note that triple arrays constructed here never coincide with Paley triple arrays. To see this, we compare the sum of the number of rows and columns. For a Paley triple array the sum is $2 q+1$, 
so it is odd. For triple arrays constructed from $(v, k, \lambda)$-difference sets with multiplier -1 the sum is $k+(v-k)=v$, which is even by Theorem 4.1. The following theorem excludes many well-known difference sets.

Theorem 4.2 (cf. [3]). Minus one is never a multiplier of a nontrivial cyclic difference set.

Thus we turn our attention to noncyclic groups. Kibler [6] gave a list of the small noncyclic $(v, k, \lambda)$ difference sets for $k<20$ up to equivalence. This list consists of 74 difference sets in 37 groups. But the only groups here satisfying the necessary conditions of being both abelian and of even order are of order 16 or 36 .

The case $(v, k, \lambda)=(16,6,2)$

Here there are 12 groups having a total of 27 difference sets. Four of these groups are abelian, having a total of eight difference sets. But only four difference sets admit -1 as multiplier. These groups and sets were pointed out in [7] and are given here below with numbering as in [6].

(B) Abelian. $\left\langle a, b: a^{4}=b^{4}=1\right\rangle$

3. $\left\{1, a, a^{2}, b, a b^{2}, a^{2} b^{3}\right\}$

5. $\left\{1, a, b, a^{2} b, a b^{2}, a^{2} b^{2}\right\}$

(C) Abelian. $\left\langle a, b, c: a^{4}=b^{2}=c^{2}=1\right\rangle$

7. $\left\{1, a, a^{2}, a b, a c, a^{3} b c\right\}$

(D) Abelian. $\left\langle a, b, c, d: a^{2}=b^{2}=c^{2}=d^{2}=1\right\rangle$

8. $\{1, a, b, c, d, a b c d\}$

The case $(v, k, \lambda)=(36,15,6)$

Here there are nine groups having a total of 34 difference sets. The two abelian groups contain seven difference sets, but only the following admits -1 as multiplier.

(A II) Abelian. $\left\langle a, b, c, d: a^{3}=b^{3}=c^{2}=d^{2}=1\right\rangle$

17. $\left\{1, a, a^{2}, c, a^{2} c, b c, a^{2} b c, b^{2} c, a^{2} b^{2} c, a d, a^{2} b d, b^{2} d, a c d, b c d, a^{2} b^{2} c d\right\}$

Applying Constructions 3.1 and 3.2 to the difference sets above give $6 \times 10$ and $15 \times 21$ triple arrays by Theorem 3.4. We note that these difference sets are all members of the following well-known and important family.

Definition 4.3. A $\left(4 u^{2}, 2 u^{2} \pm u, u^{2} \pm u\right)$-difference sets is called a Hadamard difference set.

To our knowledge, almost all reversible difference sets are Hadamard. This question was systematically studied by [12] and [9], and the only exception known is due to McFarland [10] who proved there is an abelian reversible $(4000,775,150)$-difference set and proposed the following conjecture.

Conjecture 4.4 (McFarland's Conjecture). If $D$ is a reversible difference set, then either $D$ is a (4000, 775, 150)-difference set or $D$ is a Hadamard difference set.

For Hadamard difference sets there is a strong nonexistence theorem.

Theorem 4.5. [11] If there exists a Hadamard difference set with multiplier minus one in an abelian group of order $4 u^{2}$, then the square-free part of $u$ can only have the prime factors 2 and 3 .

There are several constructions of reversible difference sets, and they were put together in [8] to give the following result. 
Theorem 4.6 (cf. [4]). Let $G$ be a group of the form $G=\mathbb{Z}_{4}^{b} \times \mathbb{Z}_{2^{c_{1}}}^{2} \times \ldots \times \mathbb{Z}_{2^{c_{r}}}^{2}$, and let $\boldsymbol{H}$ be a group of the form $H=\mathbb{Z}_{2}^{2} \times \mathbb{Z}_{3}^{2 a} \times \mathbb{Z}_{p_{1}}^{4} \times \ldots \times \mathbb{Z}_{p_{s}}^{4}$, where the $p_{j}$ are (not necessarily distinct) odd primes and where $a, b, c_{1}, \ldots, c_{r}$ are nonnegative integers. Then both $G$ and $G \times H$ contain reversible Hadamard difference sets.

In [4] Theorems 4.5 and 4.6 are combined to give the following.

Corollary 4.7 (cf. [4]). Let $u$ be a positive integer. There exists a reversible Hadamard difference set in some abelian group of order $4 u^{2}$ if and only if the square-free part of $u$ divides 6.

We conclude this section by giving an existence result for triple arrays based on the knowledge of abelian reversible difference sets.

Theorem 4.8. Let $u$ be a positive integer such that the square-free part of $u$ divides 6, then there is a TA $\left(4 u^{2}-1, u^{2}, u^{2}+u, u^{2}-u, u^{2}:\left(2 u^{2}-u\right) \times\left(2 u^{2}+u\right)\right)$. Moreover, there is a $T A(3999,625,2600,150,625: 775 \times 3225)$.

Proof. By Corollary 4.7 we know that there is an abelian reversible $\left(4 u^{2}, 2 u^{2}-u, u^{2}-u\right)$-difference sets when the square-free part of $u$ divides 6 , and by [10] that there is an abelian reversible (4000, 775, 150)-difference set. Applying Theorem 3.4 to these two cases gives the existence of the corresponding triple arrays, where the parameters are calculated using Theorem 1.6 and Corollary 2.14. $\square$

\section{5 | A DIRECT CONSTRUCTION}

To help analyze non-abelian difference sets, we give a more direct construction of row-column designs from difference sets. This is nothing new: we have simply rewritten the previous construction leaving out the intermediate step via Youden squares.

Definition 5.1. Let $D$ be a nonempty proper subset of $G$. Construct an array $A(G, D)$ with rows indexed by $D$ and columns by $G \backslash D$, with the $(x, y)$ entry equal to $x^{-1} y$. Note that the entries of the array are nonidentity elements of $G$, and that no element of $G$ is repeated in a row or column.

Proposition 5.2. Suppose that $D$ is a left difference set in a group $G$ of order $v$. Then $A(G, D)$ is a double array of size $k \times(v-k)$ on $v-1$ symbols. It is a triple array if and only if the size of $x^{-1} D \cap D^{(-1)} y$ is constant for $x \in D, y \in G \backslash D$.

Proof. Suppose that $D$ is a $(v, k, \lambda)$ left difference set, so that any two left translates of $D$ meet in $\lambda$ elements. The cancelation law for groups shows that no element is repeated in a row or column; and clearly the identity does not occur anywhere in the array. Given $z \neq 0$, there are $k$ representations as $z=x^{-1} y$ with $x \in D$ (one for each element of $D$; and $\lambda$ of these have $y \in D$. So the number of occurrences of $z$ in the table is $k-\lambda$. Further, we see that

- row $x$ of $A$ contains the elements of $x^{-1}(G \backslash D)$;

- column $y$ of $A$ contains the elements of $D^{(-1)} y$.

If $D$ is a left difference set, then so is $G \backslash D$. Moreover, $D^{(-1)} y=\left(y^{-1} D\right)^{-1}$, so any two translates of $D^{(-1)}$ meet in a constant number of points. It follows immediately that $A$ is a double array. The condition for $A$ to be a triple array is clear.

Suppose that $D^{(-1)}$ is equal to a left translate of $D$, and that every right translate of $D$ is also a left translate. Then any row and any column contain a constant number of common elements, so we have 
T A B L E $1 \quad(16,6,2)$ non-abelian difference sets

\begin{tabular}{|lllll|}
\hline Group & Difference set & Reversible & Weak mult $\mathbf{- 1}$ & Left $=$ Right \\
\hline E & 9 & 8 & 8 & 8 \\
\hline F & 10 & 8 & 8 & 8 \\
\hline & 11 & - & - & 4 \\
\hline G & 12 & - & 8 & 8 \\
\hline H & 13 & - & - & 8 \\
\hline & 14 & - & - & 4 \\
\hline & 15 & 4 & 8 & 8 \\
\hline I & 16 & 4 & 8 & 8 \\
\hline & 17 & 4 & 8 & 8 \\
\hline J* & 18 & 4 & 8 & 8 \\
\hline & 19 & - & 8 & 8 \\
\hline K & 20 & 4 & 8 & 8 \\
\hline & 21 & - & 8 & 8 \\
\hline L & 22 & 4 & 8 & 8 \\
\hline & 23 & 4 & 8 & 4 \\
\hline
\end{tabular}

a triple array. Thus, if $D$ is a difference set with multiplier -1 in an abelian group, then $A$ is a triple array.

Any triple array with $v=r+c-1$ gives a SBIBD, as Agrawal pointed out. The points of the design are the rows and columns of the array (in the first form we described); the blocks are indexed by the letters and one additional symbol $\star$, where $\star$ is incident with all rows and no columns, while a letter is incident with the rows not containing it and the columns containing it.

For triple arrays from difference sets with multiplier -1 , one can ask whether this design coincides with the one obtained by developing the difference set. It is immediate from our direct construction in this section that the answer is yes. For the difference set $D$ is a block of the design, and we have given bijections between the rows and $D$, and between the columns and $G \backslash D$; the two bullet points in the proof of Proposition 5.2 show that the designs coincide.

\section{6 | NON-ABELIAN DIFFERENCE SETS}

Question 6.1. Suppose that $D$ is a left difference set in a non-abelian group $G$. Is it ever possible for $A(G, D)$ to be a triple array?

We next discuss some computations related to this question. (We have not found any examples.)

Proposition 6.2. Let $D$ be a left difference set in a group $G$. Then

(a) Denerates $G$, so $D$ cannot be contained in a proper subgroup of $G$.

(b) The set $S=\{x \in G: x D=D y$ for some $y \in G\}$ is a subgroup of $G$.

(c) If $D \subseteq S$, then $S=G$. 
T A B L E $2 \quad(36,15,6)$ non-abelian difference sets

\begin{tabular}{|c|c|c|c|c|}
\hline Group & Difference set & Reversible & Weak mult -1 & Left $=$ Right \\
\hline \multirow[t]{6}{*}{ IB } & 5 & 2 & 6 & 6 \\
\hline & 6 & - & - & 6 \\
\hline & 7 & - & - & 6 \\
\hline & 8 & 2 & 6 & 6 \\
\hline & 9 & - & - & 6 \\
\hline & 10 & - & - & 6 \\
\hline IC & 11 & - & - & 4 \\
\hline \multirow[t]{5}{*}{ ID } & 12 & 3 & 9 & 9 \\
\hline & 13 & 3 & 9 & 9 \\
\hline & 14 & - & - & 9 \\
\hline & 15 & 6 & 18 & 18 \\
\hline & 16 & 2 & 2 & 2 \\
\hline IIB & 20 & 4 & 4 & 4 \\
\hline \multirow[t]{6}{*}{ IIC } & 21 & - & - & 6 \\
\hline & 22 & 2 & 6 & 6 \\
\hline & 23 & 2 & 6 & 6 \\
\hline & 24 & - & - & 6 \\
\hline & 25 & - & - & 6 \\
\hline & 26 & 2 & 6 & 6 \\
\hline \multirow[t]{6}{*}{ IID } & 27 & 3 & 3 & 3 \\
\hline & 28 & 4 & 6 & 6 \\
\hline & 29 & 3 & 3 & 3 \\
\hline & 30 & 4 & 6 & 6 \\
\hline & 31 & 4 & 6 & 6 \\
\hline & 32 & 3 & 3 & 3 \\
\hline \multirow[t]{2}{*}{ IIE } & 33 & - & - & 3 \\
\hline & 34 & 1 & 3 & 3 \\
\hline
\end{tabular}

Proof. (a) holds because every element of $G$ has the form $x^{-1} y$ for some $x, y \in D$.

To see that (b) holds, note that $G \times G$ acts on the power set of $G$ by the diagonal action $(x, y)$ : $D \mapsto x^{-1} D y$; the stabilizer of a set $D$ is thus a subgroup of $G \times G$, and the set $S$ of the Proposition is the projection of this subgroup onto the first factor.

Before we discuss the non-abelian difference sets in Kibler's list [7], we recall some preliminary observations.

(a) $D$ is a left difference set if and only if it is a right difference set (that is, the nonidentity elements of $G$ occur equally often in the form $y x^{-1}$, for $x, y \in D$ [5].

(b) We say that -1 is a weak multiplier of $D$ if the set $D^{(-1)}=\left\{d^{-1}: d \in D\right\}$ (which is also a difference set) is a translate $D x$ of $D$, for some $x \in G$.

(c) We say that $D$ is reversible if $D^{(-1)}=D$. Note that, if $D^{(-1)}=D x^{2}$ where $x$ is central in $G$, then

$$
(D x)^{(-1)}=(x D)^{(-1)}=D^{(-1)} x^{-1}=D x,
$$

so $D x$ is a reversible difference set. 
The case $(v, k, \lambda)=(16,6,2)$

For this case Kibler found, up to equivalence, that eight of the 12 groups having difference sets are non-abelian, containing 19 difference sets.

Note that Kibler's group $(\mathrm{J})$ is incorrect. The presentation of the group should be $\left\langle a, b: a^{8}=b^{2}=\right.$ $\left.1, b a=a^{5} b\right\rangle$, and the two difference sets are $\left\{1, a, a^{2}, a^{5}, a^{4} b, a^{2} b\right\}$ and $\left\{1, a, a^{3}, a^{4}, a^{3} b, a^{5} b\right\}$. This is noted by an asterisk in the table below. The other notation is as in Kibler's paper.

Using GAP [22], we examined all cases. Data on the number of reversible translates of $D$, the number of translates with a weak multiplier -1 (this is weaker than having a reversible translate), and on the number $s$ of $x$ for which $x D=D y$ for some $y$, is given in Table 1. Each of these numbers is independent of the particular choice of difference set among the collection of all translates.

Note that $s=v$ would be equivalent to the assertion that every left translate is a right translate (giving a triple array); but this never occurs.

Table 2 has the same information for the non-abelian $(36,15,6)$ difference sets. Again, we fail to find a triple array.

\section{REFERENCES}

[1] H. Agrawal, Some methods of construction of designs for two-way elimination of heterogeneity, J. Amer. Statist. Assoc. 61 (1966), 1153-1171.

[2] S. Bagchi, On two-way designs, Graphs Combin. 14 (4) (1998), 313-319.

[3] M. K. Baumert, Cyclic difference sets, Springer-Verlag, New York, Lecture Notes in Mathematics, 1971.

[4] T. Beth, D. Jungnickel, and H. Lenz, Design theory, (2nd ed. Cambridge University Press, Cambridge, England, 1999.

[5] R. H. Bruck, Difference sets in a finite group, Trans. Amer. Math. Soc. 78 (1955), 464-481.

[6] R. E. Kibler, A summary of noncyclic difference sets, $k<20$, J. Combin. Theory (A) 25 (1978), 62-67.

[7] E. C. Johnsen, The inverse multiplier for abelian group difference sets, Canad. J. Math. 16 (1964), 787-796.

[8] D. Jungnickel and B. Schmidt, Difference sets: An update, In: Geometry, combinatorial designs and related structures, J. W. P. Hirschfeld, S. S. Magliveras, and M. J. de Resmini (eds.), Cambridge University Press, Cambridge, 1999, pp. 89-112.

[9] S. L. Ma, McFarland's conjecture on abelian difference sets with multiplier-1, Designs, Codes Cryptogr. 1(1991), 321-332.

[10] R. L. McFarland, A family of difference sets in non-cyclic groups, J. Comb. Th. (A) 15 (1973), 1-10.

[11] R. L. McFarland, Subdifference sets of Hadamard difference sets, J. Comb. Th. (A) 54 (1990), 112-122.

[12] R. L. McFarland and S. L. Ma, Abelian difference sets with multiplier-1, Arch. Math. 54 (1990), 610-623.

[13] J. P. McSorley, Double arrays, triple arrays, and balanced grids withv $=r+c-1$, Designs Codes Cryptogr. 37 (2005), 313-318.

[14] J. P. McSorley, N. C. K. Phillips, W. D. Wallis, and J. L. Yucas, Double arrays, triple arrays and balanced grids, Designs Codes Cryptogr. 35 (2005), 21-45.

[15] E. H. Moore and H. S. Pollatsek, Difference sets: Connecting algebra, combinatorics, and geometry, Am. Math. Soc. 2013.

[16] T. Nilson and L.-D. Öhman, Triple arrays and Youden squares, Designs Codes Cryptogr. 75 (3) (2015), 429451.

[17] D. A. Preece, W. D. Wallis, and J. L. Yucas, Paley triple arrays, Australas J. Combin. 33 (2005), $237-246$.

[18] D. Raghavarao and G. Nageswararao, A note on a method of construction of designs for two-way elimination of heterogeneity, Commun. Stat. 3 (2) (1974), 197-199.

[19] J. R. Seberry, A note on orthogonal Graeco-Latin designs, Ars. Combin. 8 (1979), 85-94. 
[20] C. A. B. Smith and H. O. Hartley, The Construction of Youden squares, J. Stat. Soc. S B Methodol. 10 (2) (1948), $262-263$.

[21] D. J. Street, Graeco-Latin and nested row and column designs, Combin. Math., Lecture Notes Math. 884, Springer 8 (1981), 304-313.

[22] The GAP Group, GAP - Groups, algorithms, and programming, Version 4.7.2; 2013: http://www.gap-system.org.

[23] W. D. Wallis and J. L. Yucas, Note on Agrawal's “Designs for Two-way Elimination of Heterogeneity,” J. Combin. Math. Combin. Comput. 46 (2003), 155-160.

How to cite this article: Nilson T, Cameron Peter J. Triple arrays from difference sets. $J$. Combin. Design. 2017;25:494-506. https://doi.org/10.1002/jcd.21569 